\title{
Indication of family-specific DNA methylation patterns in developing oysters
}

Claire E. Olson and Steven B. Roberts

School of Aquatic and Fishery Sciences, University of Washington, Seattle, WA, United

States

Submitting Author : Claire E. Olson, University of Washington, School of Aquatic and Fishery Sciences, 1122 Boat Street, Seattle, WA 98105-5020, United States. email: che625@uw.edu

Corresponding Author : Steven B. Roberts, University of Washington, School of Aquatic and Fishery Sciences, 1122 Boat Street, Seattle, WA 98105-5020, United States. email: sr320@uw.edu 


\begin{abstract}
Background

DNA methylation is an epigenetic modification that is ubiquitous across many eukaryotes, with variable patterns and functions across taxa. The roles of DNA methylation during invertebrate development remain enigmatic, especially regarding the inheritance and ontogenetic dynamics of methylation. In order to better understand to what degree DNA methylation patterns are heritable, variable between individuals, and changing during Crassostrea gigas development, we characterized the genome-wide methylome of Crassostrea gigas sperm and larvae from two full-sib families nested within a maternal half-sib family across developmental stages.
\end{abstract}

\title{
Results
}

Bisulfite treated DNA sequencing of Crassostrea gigas sperm and larvae at 72 hours post fertilization and 120 hours post fertilization revealed DNA methylation ranges from $15-18 \%$. Our data suggest that DNA methylation patterns are inherited, as methylation patterns were more similar between the two sires and their offspring compared to methylations pattern differences among developmental stages. Loci differing between the two paternal full-sib families (189) were found throughout the genome but were concentrated in transposable elements. The proportion of differentially methylated loci among developmental stages was not significantly greater in any genomic region. 


\section{Conclusions}

This study provides the first single-base pair resolution DNA methylomes for both oyster sperm and larval samples from multiple crosses. Assuming DNA methylation is introduced randomly, the predominance of differentially methylated loci between families within transposable elements could be associated with selection against altering methylation in gene bodies. For instance, differentially methylated loci in gene bodies could be lethal or deleterious, as they would alter gene expression. Another possibility is that differentially methylated loci may provide advantageous phenotypic variation by increasing transposable element mobility. Future research should focus on the relationship between epigenetic and genetic variation, and explore the possible relationship of DNA methylation and transposable element activity. 


\section{Introduction}

DNA methylation is an epigenetic modification that is ubiquitous across many eukaryotes, with variable patterns and functions across taxa. This epigenetic mechanism involves the addition of a methyl group to cytosines, usually in $\mathrm{CpG}$ dinucleotides, catalyzed by DNA methyltransferases. Epigenetic modifications such as DNA methylation can alter gene expression without modifying the underlying nucleotide sequence, and functions in mammals to suppress transcription through increased methylation in promoter regions (Bell and Felsenfeld, 2000). In mammals, DNA methylation is essential for development and differentiation of organs and tissues (Okano et al. 1999). Likewise, mutations of DNA methyltransferase in mammals may result in developmental delays and mortality (Li et al. 1992).

In contrast to the densely methylated mammalian genomes, several invertebrate species display low to intermediate levels of methylation. In invertebrates, it has been proposed that DNA methylation of genes may be associated with alternative splicing events (i.e. honey bee (Lyko et al. 2010) and Nasonia (Park et al. 2011)). Methylation of gene bodies has also been shown to have a positive relationship with transcriptional activity in oysters (Gavery and Roberts, 2013; Olson and Roberts, 2014a). Currently there is an incomplete understanding of the regulation of gene expression by DNA methylation in invertebrates, though it appears to be distinct from mechanisms observed in mammals and likely varies across species.

From the limited studies that have focused on invertebrates, research has shown that similar to mammals, DNA methylation has important regulatory functions during early development. For example, research on the honey bee Apis mellifera found DNA 
methylation to be abundant in the genome, with methylation being associated with altered gene expression resulting in bee caste differentiation (Elango et al. 2009; Kucharski et al. 2008). Furthermore, DNA methylation has been shown to regulate gene expression during Octopus vulgaris development, particularly during the first paralarval stage that includes significant morphological changes (Diaz-Freije et al. 2014). The first indication that methylation was an important regulator of development in $C$. gigas was by Riviere et al. (2013), who found treatment with 5-Aza-cytidine leads to developmental alterations and abnormal phenotypes in oysters.

Despite the essential role of methylation in development, there is limited information on individual variation in DNA methylation patterns among invertebrates and particularly how any methylation information might be passed on to offspring. Furthermore, we do not have a full understanding of ontogenetic changes in DNA methylation. In order to better understand to what degree DNA methylation patterns are heritable, variable between individuals, and changing during $C$. gigas development, we analyzed genome-wide DNA methylation in gametes and larval oysters (72 and 120 hours post-fertilization) from two full-sib families.

\section{Methods}

\section{Experimental Design}

Oysters (two males and a single female) were collected from Oakland Bay, South Puget Sound, WA. No ethical approval or permits were required to work with this species. Oysters were strip spawned by scoring the gonad tissue with a sterile razor blade and rinsing out gametes with sterile seawater. Oocytes were incubated for 30 minutes in 
sterile seawater and 2 million oocytes each were placed into two separate plastic containers. Sperm diluted in sterile seawater (1L) from each male were used to fertilize oocytes. Fertilization was confirmed by examining polar bodies in cells under a compound microscope.

Larvae were kept in static tanks (100L) up to 120 hours post-fertilization (hpf). Counts of oyster larvae were performed at $120 \mathrm{hpf}$ to confirm normal development. Two samples for DNA methylation analyses were taken from sperm prior to fertilization, and larvae samples collected at days $72 \mathrm{hpf}$ and $120 \mathrm{hpf}$. Larvae samples were taken by filtering on a $20 \mu \mathrm{m}$ screen. All samples were preserved in $95 \%$ ethanol. For simplicity the sperm and corresponding larvae samples are referred to as family \#1 and family \#3 based on paternity.

\section{Bisulfite treated DNA Sequencing (BS-Seq)}

Genomic DNA was extracted using DNAzol according to the manufacturer's protocol (Molecular Research Center, Inc., Cincinnati, OH). High molecular weight genomic DNA (6 ug per sample), was used to prepare six libraries for whole-genome bisulfite sequencing. Briefly, DNA was fragmented to an average length of $250 \mathrm{bp}$ in an Adaptive Focused Acoustics (AFA) microtube using a Covaris S2 (Covaris Inc Woburn, MA) with the following settings: duty cycle $20 \%$, intensity of 4.0 , cycles per burst 200 , for 60 seconds. Fragment size resulting from DNA shearing was confirmed by gel electrophoresis. Libraries were constructed using the Paired-End DNA Sample Prep Kit (Illumina, San Diego, CA) with standard protocols. Unmethylated Lambda DNA (0.5\%) (Promega Co. Madison, WI) was added to the each sample prior to fragmentation and 
library construction to serve as a measure of bisulfite conversion efficiency. DNA was treated with sodium bisulfite using the EpiTect Bisulfite Kit (Qiagen, Valencia, CA) and 72 bp paired-end sequencing was performed on the Illumina HiSeq 2000 system. Library construction and sequencing was performed by the High Throughput Genomics Center (htSEQ, Seattle, WA).

Bisulfite sequencing reads from the six libraries were quality filtered to remove adapter sequences and separately mapped to the Crassostrea gigas genome (version GCA_000297895.1; Zhang et al. 2012) using Bisulfite Sequencing Mapping Program BSMAP v2.74 (Xi and Li 2009). Resulting alignment from mapping bisulfite treated reads was analyzed with methratio, a Python script that accompanies BSMAP. Parameters for methratio included reporting loci with zero methylation ratios (-z), combining $\mathrm{CpG}$ methylation ratios on both strands (-g) and only using unique mappings (-u). Only CpG loci covered by at least 3 sequenced reads were considered for further analysis. Data can be accessed and computational analysis performed as described via a GitHub repository (Olson and Roberts, 2014b). The IPython notebook in this repository includes all steps necessary for downloading and reproducing the analyses described in this manuscript.

\section{Global DNA Methylation Comparison}

Whole-genome DNA methylation correlation and clustering were performed using the program methylKit 0.9.2 (Akalin et al. 2012) in R v3.0.3. Pairwise Pearson's correlation coefficients scores were calculated based on the percent methylation profiles 
between all pairs of samples. Hierarchical clustering was performed using 1-Pearson's correlation distance of the six methylation profiles.

\section{Differentially Methylated Loci}

Differential methylation between the two full-sib families at each locus was determined using Fisher's exact test in methylKit. A CpG locus was determined to be different between families when the difference in methylation ratio between families was more than $25 \%$ and $p$-value $<0.01$.

Ontogenetic changes in DNA methylation patterns were tested by three pairwise comparisons (Fisher's exact tests in methylKit) between all developmental stages (sperm and $72 \mathrm{hpf}$ larvae, sperm and $120 \mathrm{hpf}$ larvae, and $72 \mathrm{hpf}$ and $120 \mathrm{hpf}$ larvae).

Differentially methylated loci were identified as any $\mathrm{CpG}$ with greater than $25 \%$ and $p$ value $<0.01$ for any comparison.

All loci with significantly different methylation ratios across families and ontogenetic stages were characterized with respect to genomic features (intron, exon, promoter region, transposable element) using Bedtools (i.e., intersectBed) (Quinlan and Hall, 2010). Genomic features were developed and reported elsewhere (Gavery and Roberts 2013). Putative promoters were defined as $1 \mathrm{~kb}$ regions upstream from transcription start sites. Transposable elements were identified using RepeatMasker, a program that screens and annotates interspersed repeats (Smit et al., 1996-2010). Specifically, RepeatProteinMask, was used with Repbase (Jurka et al. 2005), which contained 7,445 sequences. Sequence similarities from RepeatProteinMask were examined using WU-Blast (Lopez et al., 2003) to identify transposable elements which were included in the genome feature track. A total of 58,468 transposable elements 
identified based on sequence similarity. This genome feature track along with intron, exon, and promoter region feature track are all available via the IPython notebook that provides code and data used in this manuscript (Olson and Roberts, 2014b). A Chisquared test was performed to determine if the distribution of differentially methylated loci among genomic regions (intron, exon, promoter region, transposable elements) was significantly different to the distribution of all CpGs in the oyster genome.

\section{Results}

\section{Bisulfite treated DNA Sequencing (BS-Seq)}

Bisulfite treated DNA sequence reads are available (NCBI Sequence Read Archive: Study Accession Number SRP028178 - Accession Numbers SRX795174SRX795179). Sodium bisulfite conversion efficiency was estimated to be $99.9 \%$ based on analysis of lambda phage DNA. The number of sequenced cytosines ranged from $2.6 \times 10^{7}$ to $5.3 \times 10^{7}$ across libraries. Using a $3 \times$ coverage threshold, most cytosines $(75-$ $78 \%$ ) were determined to be unmethylated (methylation ratio $=0$ ), while $15-18 \%$ of the CpG dinucleotides were methylated (methylation ratio 0.5) (data not shown).

\section{Genome-wide DNA Methylation Comparison}

Relationships on a genome-wide scale were assessed by sample correlation and clustering. A total of 40,654 common loci were shared among all six samples and thus analyzed. Methylation ratios were highly correlated between sperm and respective progeny, with a pair-wise Pearson's correlation coefficient $(r)$ of 0.84 for both families. These similarities within families are also evident in hierarchical clustering (Figure 1), 
where both male gamete samples were more similar in their methylation profiles to their respective $120 \mathrm{hpf}$ larvae.

\section{Family-specific and Developmental Differences}

A total of 189 differentially methylated loci (DMLs) were identified between the two full-sib families. Of these, 99 were found to overlap with a defined genomic region (exon, intron, promoter region, transposable element) (Figure 2). Most $\mathrm{CpG}$ loci with different methylation ratios among oyster families were in introns. However, compared to the distribution of $\mathrm{CpG}$ dinucleotides in the oyster genome, the proportion of differentially methylated loci within transposable elements was significantly higher than expected $\left(\chi^{2}=22.17, \mathrm{df}=1, \mathrm{p}<0.0001\right)$

A total of $160 \mathrm{CpG}$ loci showed differences in methylation ratios among developmental stages. Of these loci, 90 were within defined genomic regions (Figure 2). The proportion of differentially methylated loci was not significantly greater in any genomic region analyzed.

\section{Discussion}

This study provides the first single-base pair resolution DNA methylomes for both oyster sperm and larval samples from multiple crosses. This research not only provides new information on DNA methylation patterns during oyster development, but also examines its inheritance and changes during early development. Interestingly, our research indicates that epigenetic patterns may differ among oyster families.

Methylation levels in oyster sperm and larvae samples ranged from 15-18\% with interspersed regions of both methylated and unmethylated DNA in both male gamete and 
larval samples. This proportion of $\mathrm{CpG}$ methylation falls within the range of that previously reported for oyster male gonad tissue (Olson and Roberts, 2014a) and oyster gill tissue (Gavery and Roberts, 2013). Overall methylation levels are also comparable to those reported among multiple developmental stages of the Pearl oyster Pinctada fucata (Li et al. 2014). These findings indicate that overall genome methylation in C. gigas is at an intermediate level and suggests that DNA methylation levels do not significantly vary between multiple cell types and life history stages. This is similar to what has been described in global 5-methylcytosine content during different stages of Ciona intestinalis development (Suzuki et al. 2013). However, it contrasts with research on mammals and vertebrates, which exhibit the presence of tissue and developmental stage specific methylation profiles, as that seen in zebrafish (McGaughey et al. 2014).

Genome-wide comparisons indicated higher similarity of methylation patterns between oyster families than between developmental stages, suggesting that DNA methylation patterns are inherited. If epigenetic marks are indeed heritable, this mechanism has significant implications for selection. It has been proposed that epigenetic variation may compensate for a decrease in genetic variation in species such as sparrows (Shrey et al. 2012). While outside the scope of the current study, an assessment of relationships between genetic and epigenetic variation is critical. Several studies have examined epigenetic differentiation in vertebrate and plant populations experiencing different environments, indicating evidence for divergent selection in these species (Liu et al. 2012; Herrera and Bazaga 2010). Few studies have focused on invertebrates, though Jiang et al. (2013a) investigated the relationship between genetic and epigenetic variations in two groups of $C$. gigas, a base stock domesticated population and third 
generation mass selection population. This study demonstrated genetic differentiation between the base population and third generation mass selection populations of oysters, but did not find overall epigenetic variation (Jiang et al. 2013a). Nevertheless, a significant correlation was observed between genetic and epigenetic profiles, with few individuals having similar genetic but distinct epigenetic profiles (Jiang et al. 2013a). Regardless, if epigenetic variation is independent of genotype, mechanisms involved in epigenetic inheritance are not fully understood.

Differences in genome-wide methylation ratios between full-sib families nested within a maternal half sib family suggested paternal inheritance of DNA methylation patterns. These results are similar to what has been seen in zebrafish where embryos inherit the methylation profile of sperm rather than oocyte (Jiang et al. 2013b, Potok et al. 2013). In contrast, methylation ratios in Pearl oysters are mainly influenced by oocytes, rather than sperm (Li et al. 2014), possibly due to maternal influences on DNA methylation patterns in early larvae, while later stages attain methylation patterns similar to sperm.

Differentially methylated loci across families were distributed throughout the genome, though a higher proportion was found in transposable elements. This concentration of methylation in transposable elements may be due to selection against altering methylation in functionally important parts of the genome. For instance, many differentially methylated loci in gene bodies could be lethal or deleterious as they would alter gene expression. It should be noted that the role of DNA methylation in regulating genome activity in C. gigas is still unclear. However, it has been suggested that elevated methylation decreases spurious transcription of housekeeping genes and limited 
methylation in inducible genes facilitates multiple transcriptional opportunities (Roberts and Gavery, 2012). In other words, DNA methylation patterns in gene bodies may have evolved over time based on gene function to fit the needs of organisms in highly variable environments, and random changes in these patterns could be detrimental. Furthermore, we suggest that random variations in methylation within transposable elements may have a relatively higher chance of persisting than elsewhere in the genome. Transposable elements are mobile DNA sequences that may be methylated in many species to silence activity (Yoder et al. 1997; Liu and Schmid 1993). Limited information is available about the methylation status of transposable elements in other invertebrate species, but the available studies suggest that transposons are generally unmethylated and contain similar levels of methylation to neighboring DNA (Suzuki and Bird, 2008). This is in agreement with our previous research, which showed limited DNA methylation in transposable elements in oyster male gamete tissue (Olson and Roberts, 2014a). Assuming that transposable element activity is less critical to survival than coding gene activity, differentially methylated loci in transposable elements may be less likely to have negative selective effects. On the other hand, differentially methylated loci may also provide advantageous phenotypic variation by increasing transposable element mobility. However, such selection hypotheses assume that methylation is introduced randomly, something we do not have evidence for.

Interestingly, we did not observe a high proportion of differentially methylated loci among promoter regions, as would be expected if promoter methylation was regulating gene expression to play a role in oyster development. Recent research has found that DNA methylation of promoter regions specifically reduces expression of Hox 
genes during oyster development (Riviere et al. 2013). Considerable stage-specific differences in total methylation levels during oyster early development indicated that DNA methylation plays a crucial role in oyster embryogenesis (Riviere et al. 2013). We previously found variation in expression levels depending on the level of promoter region methylation (Olson and Roberts, 2014a). Surprisingly, we did not observe any dramatic differences in overall methylation levels during oyster development, nor higher methylation of promoter regions. This discrepancy is likely due to the analysis of different ontogenetic stages, as Riviere et al. (2013) examined the first 24 hours postfertilization, and our first larval sample was taken at $72 \mathrm{hpf}$. It is also possible that only a subset of genes are transcriptionally controlled via DNA methylation and our global approach masked the ability to see differences.

\section{Conclusions}

This research suggests epigenetic inheritance as DNA methylation patterns were similar between males and their offspring and differed between oyster families.

Interestingly, we found a high proportion of family-specific methylation patterns within transposable elements. Future research should focus on the relationship between epigenetic and genetic variation, and explore the possible relationship of DNA methylation and transposable element activity.

\section{Acknowledgements}

This work was supported by a National Science Foundation award (Grant Number 1158119) to SR. 


\section{Abbreviations/Definitions}

BAFA- Adaptive Focused Acoustics

BS-Seq- Bisulfite Sequencing

DMLs- Differentially Methylated Loci

hpf- hours post-fertilization

\section{Competing Interests}

The authors declare that they have no competing interests.

\section{Author Contributions}

$\mathrm{CO}$ carried out the experimental trial, molecular work, and bioinformatic analyses. $\mathrm{CO}$ and SR participated in the design of the study and drafting of the manuscript. All authors read and approved the final manuscript. 


\section{References}

Akalin A, Kormaksson M, Li S, Garrett-Bakelman FE, Figueroa, ME, Melnick A, Mason

CE. methylKit: a comprehensive R package for the analysis of genome-wide DNA

methylation profiles. Genome Biology. 2012; doi: 10.1186/gb-2012-13-10-r87.

Bell AC, Felsenfeld G. Methylation of a CTCF-dependent boundary controls imprinted expression of the Igf2 gene. Nature. 2000; doi: 10.1038/35013100.

Diaz-Freije E, Gestal C, Castellanos-Martinez S, Moran P. The role of DNA methylation on Octopus vulgaris development and their perspectives. Frontiers in Physiology. 2014; doi: 10.3389/fphys.2014.00062.

Elango N, Hunt BG, Goodisman MAD, and Yi SV. DNA methylation is widespread and associated with differential gene expression in castes of the honey bee, Apis mellifera. Proceedings of the National Academy of Sciences. 2009; doi: 10.1073/pnas.0900301106.

Gavery MR, Roberts SB. Predominant intragenic methylation is associated with gene expression characteristics in a bivalve mollusc. PeerJ. 2013; doi:10.7717/peerj.215.

Gavery MR, Roberts SB. A context dependent role for DNA methylation in bivalves. Briefings in functional genomics. 2014; doi: 10.1093/bfgp/elt054. 
Herrera CM, Bazaga P. Epigenetic differentiation and relationship to adaptive genetic divergence in discrete populations of the violet Viola cazorlensis. New Phytologist. 2010; doi: 10.1111/j.1469-8137.2010.03298.x.

Jiang Q, Li Q, Yu H, Kong LF. Genetic and epigenetic variation in mass selection populations of Pacific oyster Crassostrea gigas. Genes Genomics. 2013; doi:10.1007/s13258-013-0114-4.

Jiang L, Zhang J, Wang JJ, Wang L, Zhang L, Li G, Yang X, Ma X, Sun X, Cai J, et al. Sperm, but not oocyte, DNA methylome is inherited by zebrafish early embryos. Cell. 2013; doi: 10.1016/j.cell.2013.04.041.

Jurka J, Kapitonov VV, Pavlicek A, Klonowski P, Kohany O., Walichiewicz J. Repbase Update, a database of eukaryotic repetitive elements. Cytogentic and Genome Research. 2005; doi: 10.1159/000084979.

Kucharski R, Maleszka J, Foret S, Maleszka R. Nutritional Control of Reproductive Status in Honey bees via DNA Methylation. Science. 2008; doi:

10.1126/science. 1153069 .

Li E, Bestor TH, Jaenisch R. Targeted mutation of the DNA methyltransferase gene results in embryonic lethality. Cell. 1992; doi: 10.1016/0092-8674(92)90611-F. 
Li Y, Guan Y, He M. Analysis of DNA methylation in tissues and development stages of pearl oyster Pinctada fucata. Genes and Genomics. 2014; doi: 10.1007/s13258-0140246-1.

Liu S, Sun K, Jiang T, Ho JP, Liu B, Feng J. Natural epigenetic variation in the female great roundleaf bat (Hipposideros armiger) populations. Molecular Genetics and Genomics. 2012; doi: 10.1007/s00438-012-0704-X.

Liu WM, Schmid CW. Proposed roles for DNA methylation in Alu transcriptional repression and mutational inactivation. Nucleic Acid Resources. 1993; doi: 10.1093/nar/21.6.1351.

Lopez R, Silventoinen V, Robinson S, Kibria A, Gish W. WU-Blast2 server at the European Bioinformatics Institute. Nucleic Acids Research. 2003; doi: 10.1093/nar/gkg573.

Lyko F, Foret S, Kucharski R, Wolf S, Falckenhayn C, Maleszka R. The honey bee epigenomes: differential methylation of brain DNA in queens and workers. PLoS ONE. 2010; doi:10.1371/journal.pbio.1000506.

McGaughey DM, Abaan HO, Miller RM, Kropp PA, Brody LC. Genomics of CpG methylation in developing and developed zebrafish. Genes Genomes Genetics. 2014; doi: $10.1534 / \mathrm{g} 3.113 .009514$ 
Okano M, Bell DW, Harber DA, Li E. DNA methyltransferases Dnmt3a and Dnmt3b are essential for de novo methylation and mammalian development. Cell.1999; doi:

10.1016/S0092-8674(00)81656-6.

Olson CE, Roberts SB. Genome-wide profiling of DNA methylation and gene expression in Crassostrea gigas male gametes. Frontiers in Physiology. 2014; doi:

10.3389/fphys.2014.00224.

Olson C, Roberts S. https://github.com/che625/olson-ms-nb. GitHub. (2015).

Park J, Peng Z, Zeng J, Elango N, Park T, Wheeler D, Werren JH, Yi SV. Comparative Analyses of DNA Methylation and Sequence Evolution Using Nasonia Genomes.

Molecular Biology and Evolution. 2011; doi: 10.1093/molbev/msf168.

Potok ME, Nix DA, Parnell TJ, Cairns BR. Reprogramming the Maternal Zebrafish Genome after Fertilization to Match the Paternal Methylation Pattern. Cell. 2013; doi: 10.1016/j.cell.2013.04.030.

Quinlan AR, Hall IM. BEDTools: a flexible suite of utilities for comparing genomic features. Bioinformatics. 2010; doi:10.1093/bioinformatics/btq033. 
Riviere G, Wu GC, Fellous A, Goux D, Sourdaine P, Favrel P. DNA methylation is crucial for the early development in the oyster C. gigas. Marine Biotechnology. 2013; doi: 10.1007/s10126-013-9523-2.

Roberts SB, Gavery MR. Is there a relationship between DNA methylation and phenotypic plasticity in invertebrates? Frontiers in Physiology. 2012; doi: 10.3389/fphys.2011.00116.

Shrey AW, Coon CA, Grispo MT, Awad M, Imboma T, McCoy ED, Mushinsky HR, Richards CL, Martin LB. Epigenetic Variation May Compensate for Decreased Genetic Variation with Introductions: A Case Study Using House Sparrows (Passer domesticus) on Two Continents. Genetics Research International 2012; doi: http://dx.doi.org/10.1155/2012/979751.

Smit AFA, Hubley R, Green P. RepeatMasker Open-3.0. 1996-2010. Available online at http://www.repeatmasker.org.

Suzuki MM, Bird A. DNA methylation landscapes: provocative insights from epigenomics. Nature Reviews Genetics. 2008; doi: 10.1038/nrg2341.

Suzuki MM, Yoshinari A, Obara M, Takuno S, Shigenobu S, Sasakura Y, et al. Identical sets of methylated and nonmethylated genes in Ciona intestinalis sperm and muscle cells. Epigenetics \& Chromatin. 2013; doi: 10.1186/1756-8935-6-38. 
Xi Y, Li W. BSMAP: whole genome bisulfite sequence MAPping program. BMC

Bioinformatics. 2009; doi: 10.1186/1471-2105-10-232.

Yoder JA, Walsh CP, Bestor TH. Cytosine methylation and the ecology of intragenomic parasites. Trends in Genetics. 1997; doi: http://dx.doi.org/10.1016/S0168-

9525(97)01181-5.

Zhang G, Fang X, Guo X, Li L, Luo R, Xu F, et al. The oyster genome reveals stress adaptation and complexity of shell formation. Nature. 2012; doi: 10.1038/nature11413. 
Figure 1. Dendogram of the male sperm and oyster larvae genome-wide methylation profiles using Pearson's correlation distance. Numeric prefix refers to family.

Figure 2. Proportion of family-specific differentially methylated loci, developmentally different differentially methylated loci, and all CpGs in the oyster genome based on genomic region. The proportion of loci located within a genomic region (Intron, Exon, Promoter Region, Transposable Element) for differentially methylated loci between families $(n=99)$, differentially methylated loci among the developmental stages $(n=90)$, and all CpGs in the oyster genome $(\mathrm{n}=10035701)$ are displayed. An asterisk indicates a statistically different distribution relative to the distribution of all $\mathrm{CpGs}$ in the oyster genome. 


\section{CpG methylation clustering}

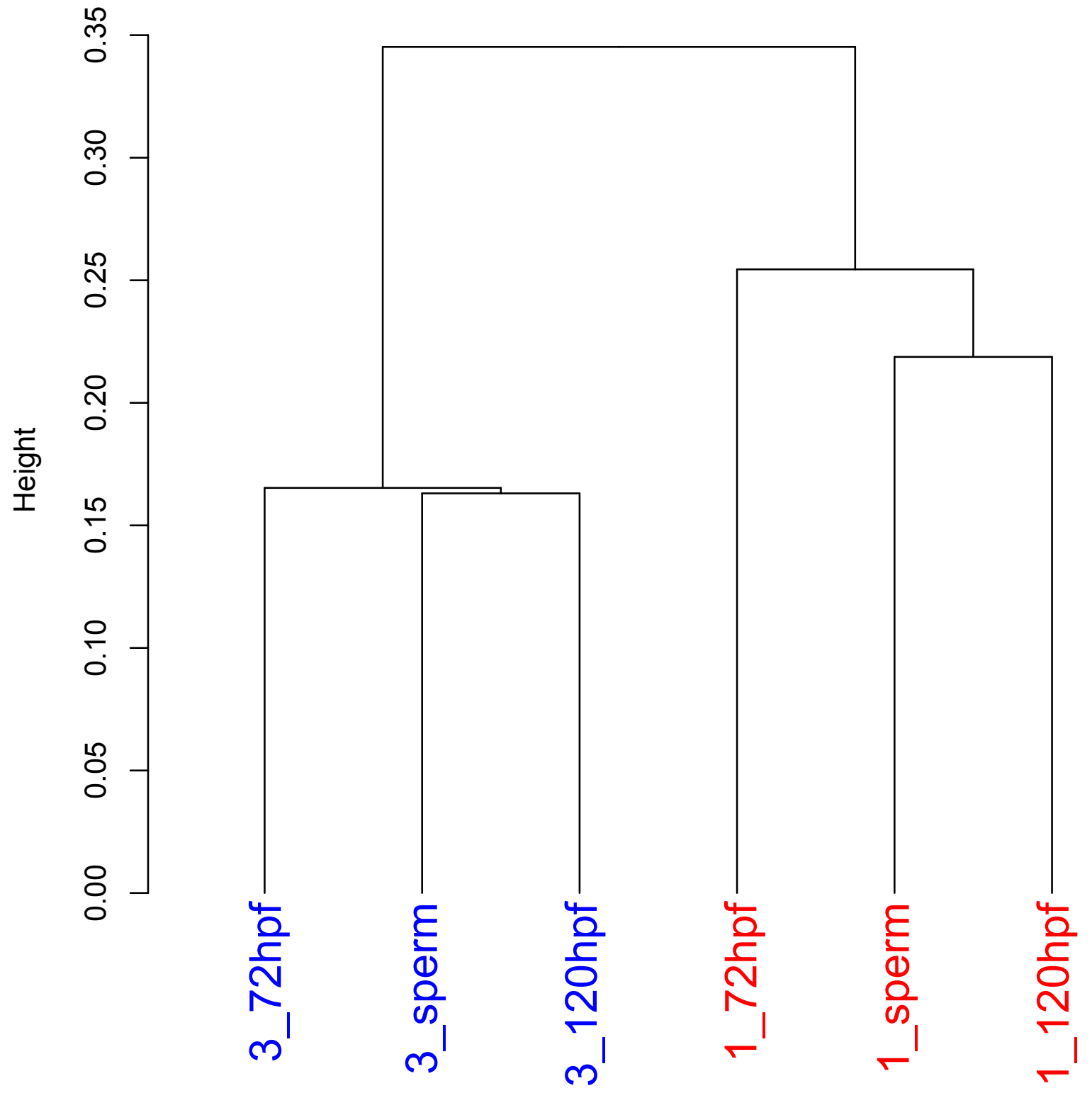




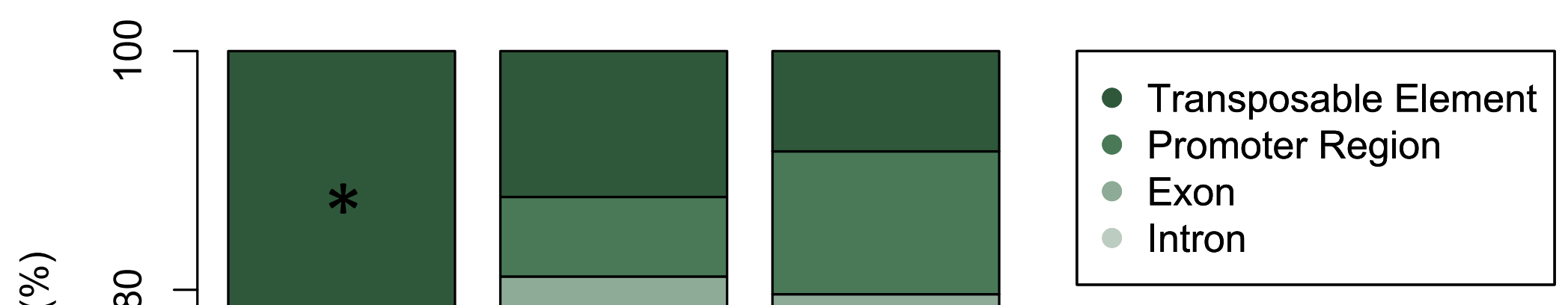

Fam DMLs Devel DMLs All CpGs 